\title{
Numerical approaches to NLO QCD calculations *
}

\author{
Giovanni Ossola \\ NCSR Demokritos, 15310, Athens, Greece \\ E-mail: ossola@inp.demokritos.gr
}

\section{Costas G. Papadopoulos}

NCSR Demokritos, 15310, Athens, Greece

E-mail: costas.papadopoulos@cern.ch

\section{Roberto Pittau}

Dipartimento di Fisica Teorica, Univ. di Torino and INFN, sez. di Torino, Italy.

E-mail: pittau@to.infn.it

We describe the recently proposed method (OPP) to extract the coefficients of the scalar oneloop integrals to any multi-particle (sub)-amplitude. Basically, no analytical information on the structure of the amplitude is needed, allowing for a purely numerical, but still algebraic, implementation of the algorithm to automatically perform one-loop calculation both in QCD and in the EW Theory. As an application of the algorithm, we give QCD one-loop results for the process $p p \rightarrow Z Z Z$ at the LHC.

8th International Symposium on Radiative Corrections (RADCOR)

October 1-5 2007

Florence, Italy

\footnotetext{
*Work supported by HEPTOOLS, MRTN-CT-2006-035505 and ALGOTOOLS, MTKD-CT-2004-014319
} †peaker. 


\section{INTRODUCTION}

In two recent papers [1], we proposed a reduction method (OPP) for arbitrary one-loop subamplitudes at the integrand level [2]. The method is based on idea of expressing the integrand of the one-loop amplitude in terms of the propagators that depends on the integration momentum. The solution of this equation can proceed in an hierarchical way, by exploiting numerically the set of kinematical equations for the integration momentum, corresponding to the so-called quadruple, triple and double cuts used in the unitarity-cut method $[3,4,5]$. The method requires a minimal information about the form of the one-loop (sub-)amplitude and therefore it is well suited for a numerical implementation. The method works for any set of internal and/or external masses, so that one is able to study the full electroweak model, without being limited to massless theories.

\section{The OPP method}

The starting point of the OPP reduction method is the general expression for the integrand of a generic $m$-point one-loop (sub-)amplitude

$$
A(\bar{q})=\frac{N(q)}{\bar{D}_{0} \bar{D}_{1} \cdots \bar{D}_{m-1}}, \quad \bar{D}_{i}=\left(\bar{q}+p_{i}\right)^{2}-m_{i}^{2}, \quad p_{0} \neq 0 .
$$

In the previous equation, we use a bar to denote objects living in $n=4+\varepsilon$ dimensions, and $\bar{q}^{2}=q^{2}+\tilde{q}^{2}$, where $\tilde{q}^{2}$ is $\varepsilon$-dimensional and $(\tilde{q} \cdot q)=0 . N(q)$ is the 4-dimensional part of the numerator function of the amplitude. If needed, the $\varepsilon$-dimensional part of the numerator should be treated separately, as explained later. $N(q)$ depends on the 4-dimensional denominators $D_{i}=$ $\left(q+p_{i}\right)^{2}-m_{i}^{2}$ as follows

$$
\begin{aligned}
N(q) & =\sum_{i_{0}<i_{1}<i_{2}<i_{3}}^{m-1}\left[d\left(i_{0} i_{1} i_{2} i_{3}\right)+\tilde{d}\left(q ; i_{0} i_{1} i_{2} i_{3}\right)\right] \prod_{i \neq i_{0}, i_{1}, i_{2}, i_{3}}^{m-1} D_{i} \\
& +\sum_{i_{0}<i_{1}<i_{2}}^{m-1}\left[c\left(i_{0} i_{1} i_{2}\right)+\tilde{c}\left(q ; i_{0} i_{1} i_{2}\right)\right] \prod_{i \neq i_{0}, i_{1}, i_{2}}^{m-1} D_{i} \\
& +\sum_{i_{0}<i_{1}}^{m-1}\left[b\left(i_{0} i_{1}\right)+\tilde{b}\left(q ; i_{0} i_{1}\right)\right] \prod_{i \neq i_{0}, i_{1}}^{m-1} D_{i} \\
& +\sum_{i_{0}}^{m-1}\left[a\left(i_{0}\right)+\tilde{a}\left(q ; i_{0}\right)\right] \prod_{i \neq i_{0}}^{m-1} D_{i} \\
& +\tilde{P}(q) \prod_{i}^{m-1} D_{i} .
\end{aligned}
$$

Inserted back in Eq. (2.1), this expression simply states the multi-pole nature of any $m$-point oneloop amplitude, that, clearly, contains a pole for any propagator in the loop, thus one has terms ranging from 1 to $m$ poles. The coefficients of the poles can be further split in two pieces. A piece that still depend on $q$ (the terms $\tilde{d}, \tilde{c}, \tilde{b}, \tilde{a}$ ), that vanishes upon integration, and a piece that do not depend on q (the terms $d, c, b, a$ ). Such a separation is always possible and the latter set of coefficients is immediately interpretable as the ensemble of the coefficients of all possible 4, 3, 2, 1-point one-loop functions contributing to the amplitude. 
Once Eq. (2.2) is established, the task of computing the one-loop amplitude is then reduced to the algebraical problem of fitting the coefficients $d, c, b, a$ by evaluating the function $N(q)$ a sufficient number of times, at different values of $q$, and then inverting the system. That can be achieved quite efficiently by singling out particular choices of $q$ such that, systematically, 4, 3, 2 or 1 among all possible denominators $D_{i}$ vanishes. Then the system of equations is solved iteratively. First one determines all possible 4-point functions, then the 3-point functions and so on. For example, calling $q_{0}^{ \pm}$the two solutions (in general complex) for which

$$
D_{0}=D_{1}=D_{2}=D_{3}=0,
$$

(there are 2 solutions because of the quadratic nature of the propagators) and since the functional form of $\tilde{d}(q ; 0123)$ is known, one directly finds the coefficient of the box diagram containing the above 4 denominators through the two simple equations

$$
N\left(q_{0}^{ \pm}\right)=\left[d(0123)+\tilde{d}\left(q_{0}^{ \pm} ; 0123\right)\right] \prod_{i \neq 0,1,2,3} D_{i}\left(q_{0}^{ \pm}\right) .
$$

This algorithm also works in the case of complex denominators, namely with complex masses. Notice that the described procedure can be performed at the amplitude level. One does not need to repeat the work for all Feynman diagrams, provided their sum is known: we just suppose to be able to compute $N(q)$ numerically.

The described procedure works in 4 dimensions. However, even when starting from a perfectly finite tensor integral, the tensor reduction may eventually lead to integrals that need to be regularized (we use dimensional regularization). Such tensors are finite, but tensor reduction iteratively leads to rank $m m$-point tensors with $1 \leq m \leq 5$, that are ultraviolet divergent when $m \leq 4$. For this reason, we introduced, in Eq. (2.1), the $d$-dimensional denominators $\bar{D}_{i}$, that differs by an amount $\tilde{q}^{2}$ from their 4-dimensional counterparts

$$
\bar{D}_{i}=D_{i}+\tilde{q}^{2} .
$$

The result of this is a mismatch in the cancellation of the $d$-dimensional denominators of Eq. (2.1) with the 4-dimensional ones of Eq. (2.2). The rational part of the amplitude, called $R_{1}$ [7], comes from such a lack of cancellation. A different source of Rational Terms, called $R_{2}$, can also be generated from the $\varepsilon$-dimensional part of $N(q)$ (that is missing in Eq. (2.1)). For the time being, it should be added by hand by looking at the analytical structure of the Feynman Diagrams or via a dedicated set of Feynman Rules. Examples on how to compute $R_{2}$ are reported in [7] and [8]. The Rational Terms $R_{1}$ are generated by the following extra integrals, introduced in [1]

$$
\begin{aligned}
\int d^{n} \bar{q} \frac{\tilde{q}^{2}}{\bar{D}_{i} \bar{D}_{j}} & =-\frac{i \pi^{2}}{2}\left[m_{i}^{2}+m_{j}^{2}-\frac{\left(p_{i}-p_{j}\right)^{2}}{3}\right]+\mathscr{O}(\varepsilon), \\
\int d^{n} \bar{q} \frac{q^{2}}{\bar{D}_{i} \bar{D}_{j} \bar{D}_{k}} & =-\frac{i \pi^{2}}{2}+\mathscr{O}(\varepsilon), \quad \int d^{n} \bar{q} \frac{\tilde{q}^{4}}{\bar{D}_{i} \bar{D}_{j} \bar{D}_{k} \bar{D}_{l}}=-\frac{i \pi^{2}}{6}+\mathscr{O}(\varepsilon) .
\end{aligned}
$$

The coefficients of the above integrals can be computed by looking at the implicit mass dependence (namely reconstructing the $\tilde{q}^{2}$ dependence) in the coefficients $d, c, b$ of the one-loop functions, once $\tilde{q}^{2}$ is reintroduced through the mass shift $m_{i}^{2} \rightarrow m_{i}^{2}-\tilde{q}^{2}$. One gets

$$
b\left(i j ; \tilde{q}^{2}\right)=b(i j)+\tilde{q}^{2} b^{(2)}(i j), \quad c\left(i j k ; \tilde{q}^{2}\right)=c(i j k)+\tilde{q}^{2} c^{(2)}(i j k) .
$$


Furthermore, by defining

$$
\mathscr{D}^{(m)}\left(q, \tilde{q}^{2}\right) \equiv \sum_{i_{0}<i_{1}<i_{2}<i_{3}}^{m-1}\left[d\left(i_{0} i_{1} i_{2} i_{3} ; \tilde{q}^{2}\right)+\tilde{d}\left(q ; i_{0} i_{1} i_{2} i_{3} ; \tilde{q}^{2}\right)\right] \prod_{i \neq i_{0}, i_{1}, i_{2}, i_{3}}^{m-1} \bar{D}_{i},
$$

the following expansion holds

$$
\mathscr{D}^{(m)}\left(q, \tilde{q}^{2}\right)=\sum_{j=2}^{m} \tilde{q}^{(2 j-4)} d^{(2 j-4)}(q),
$$

where the last coefficient is independent on $q, d^{(2 m-4)}(q)=d^{(2 m-4)}$. In practice, once the 4dimensional coefficients have been determined, one can redo the fits for different values of $\tilde{q}^{2}$, in order to determine $b^{(2)}(i j), c^{(2)}(i j k)$ and $d^{(2 m-4)}$. Such three quantities are the coefficients of the three extra scalar integrals listed in Eq. (2.6), respectively. Therefore, the OPP method allows an easy and purely numerical computation of the Rational Terms of type $R_{1}$.

\section{Cut Tools and the problem of the Numerical Inaccuracies}

A FORTRAN90 program (CutTools) implementing the OPP method can be found in [6], to which we refer for more details. We would like to stress that the only information needed by the code is the number and type of contributing propagators and the numerator function $N(q)$ (and its maximum rank). A particularly interesting feature of the OPP technique, also implemented in CutTools, is that it allows a natural numerical check of the accuracy of the whole procedure. Given the paramount importance of this issue in practical calculations, we describe it here in some detail.

During the fitting procedure to determine the coefficients, numerical inaccuracies may occur due to appearance of Gram determinants in the solutions for which 4, 3, 2 or 1 denominators vanish; due to the vanishing of some of the remaining denominators, when computed at a given solution; and to instabilities occurring when solving systems of linear equations.

In principle, each of these three sources of instabilities can be cured by performing a proper expansion around the problematic (i.e. exceptional) Phase-Space point. However, this often results in a huge amount of work that, in addition, spoils the generality of the algorithm. Furthermore, one is anyway left with the problem of choosing a separation criterion to identify the region where applying the proper expansion rather than the general algorithm.

The solution implemented in CutTools is, instead, of a purely numerical nature and relies on a unique feature of the OPP method: the fact that the reduction is performed at the integral level. In detail, the OPP reduction is obtained when, as in Eq. (2.2), the numerator function $N(q)$ is rewritten in terms of denominators. Therefore $N(q)$ computed for some arbitrary value of $q$ by using the 1. h. s. of Eq. (2.2) should always be numerically equal to the result obtained by using the expansion in the r. h. s. This is a very stringent test that is applied in CutTools for any PhaseSpace point. When, in an exceptional Phase-Space point, these two numbers differ more than a user defined quantity, the coefficients of the loop functions for that particular point are recomputed by using multi-precision routines [9] (with up to 2000 digits) contained in CutTools. Finally, one should mention that, usually, only very few points are potentially dangerous, namely exceptional, so that a limited fraction of additional $\mathrm{CPU}$ time is used to cure the numerical instabilities, therefore compensating the fact that the multi-precision routines are slower than the normal ones. 


\section{4. $p p \rightarrow Z Z Z$ at NLO}

The calculation is composed of two parts: the evaluation of virtual corrections, namely oneloop contributions obtained by adding a virtual particle to the tree-order diagrams, and corrections from the real emission of one additional massless particle from initial and final states, which is necessary in order to control and cancel infrared singularities. The virtual corrections are computed using the OPP reduction[1]. In particular, we make use of CutTools [6]. Concerning the contributions coming from real emission we used the dipole subtraction method [15] to isolate the soft and collinear divergences and checked the results using the phase space slicing method [13] with soft and collinear cutoffs, as outlined in [14].

These results have also been recently presented, following a very different approach, by Lazopoulos et al in Ref. [10]. A more complete study, that will also include the case of $W^{+} W^{-} Z$, $W^{ \pm} Z Z$, and $W^{+} W^{-} W^{ \pm}$production, will be presented in a forthcoming publication [11].

Let us begin with the evaluation of the virtual QCD corrections to the process $q \bar{q} \rightarrow Z Z Z$. We consider the process

$$
q\left(p_{1}\right)+\bar{q}\left(p_{2}\right) \longrightarrow Z\left(p_{3}\right)+Z\left(p_{4}\right)+Z\left(p_{5}\right)
$$

At the tree-level, there are six contributions to this process, obtained by permuting the final legs in all possible ways. One-loop corrections are obtained by adding a virtual gluon to the tree-level structures. Overall this calculation involves the reduction of 48 diagrams.

We perform a reduction to scalar integrals using the OPP reduction method [1]. The coefficients determined in this manner should be multiplied by the corresponding scalar integrals. Since, in the process that we are studying, no $q$-dependent massive propagator appears, we will only need massless scalar integrals. They are computed using the package OneLOop written by A. van Hameren [12].

The last step is the calculation of Rational Terms. As explained in Section 2, part of this contribution, that we call $R_{1}$, is automatically included by the to the reduction algorithm. The second term $R_{2}$, coming from the explicitly $\varepsilon$-dimensional part of the amplitude, has been added computed separately; it turns out that only three- and two-point functions contribute and the result is proportional to the tree-order amplitude.

We checked that our results, both for poles and finite parts, agree with the results obtained by the authors of Ref. [10].

In what concerns the real emission, we only have to deal with initial state singularities, where we distinguish $q \bar{q}$ and $q g$ initial states. For the $q g$ initial state, no soft singularity is present because the corresponding tree-level contribution vanishes. We recall that the structure of the NLO partonic cross sections is as follows:

$$
\begin{aligned}
& \sigma_{q \bar{q}}^{N L O}=\int_{V V V}\left[d \sigma_{q \bar{q}}^{B}+d \sigma_{q \bar{q}}^{V}+d \sigma_{q \bar{q}}^{C}+\int_{g} d \sigma_{q \bar{q}}^{A}\right]+\int_{V V V g}\left[d \sigma_{q \bar{q}}^{R}-d \sigma_{q \bar{q}}^{A}\right] \\
& \sigma_{g q}^{N L O}=\int_{V V V}\left[+d \sigma_{g q}^{C} \int_{g} d \sigma_{g q}^{A}\right]+\int_{V V V g}\left[d \sigma_{g q}^{R}-d \sigma_{g q}^{A}\right]
\end{aligned}
$$

where $d \sigma^{B}, d \sigma^{V}, d \sigma^{C}, d \sigma^{R}, d \sigma^{A}$ are respectively the Born cross section, the virtual, virtual counterterm, real and real-subtraction cross sections. For the $q \bar{q}$ initial state two dipoles are needed as 
subtraction terms. If $p_{6}$ is the momentum which can become soft or collinear, the dipole term for gluon emission off the quark is given by

$$
\begin{aligned}
\mathscr{D}^{q_{1} g_{6}, \bar{q}_{2}} & =\frac{8 \pi \alpha_{s} C_{F}}{2 \tilde{x} p_{1} \cdot p_{6}}\left(\frac{1+\tilde{x}^{2}}{1-\tilde{x}}\right)\left|\mathscr{M}_{q \bar{q}}^{B}(\{\tilde{p}\})\right|^{2} \\
\tilde{x} & =\frac{p_{1} \cdot p_{2}-p_{2} \cdot p_{6}-p_{1} \cdot p_{6}}{p_{1} \cdot p_{2}}
\end{aligned}
$$

where the $\{\tilde{p}\}$ are redefined momenta, $\left\{\tilde{p}_{j}\right\}=\left\{\tilde{p}_{16}, \tilde{p}_{2}, \tilde{p}_{3}, \tilde{p}_{4}, \tilde{p}_{5}\right\}$, which are again on-shell and go to $\left\{p_{1}, \ldots, p_{5}\right\}$ in the singular limit, e.g. $\tilde{p}_{16}=\tilde{x} p_{1}$. The regularised real emission part then reads

$$
d \sigma_{q \bar{q}}^{R}-d \sigma_{q \bar{q}}^{A}=\frac{1}{6} \frac{1}{N} \frac{1}{2 s_{12}}\left[C_{F}\left|\mathscr{M}_{q \bar{q}}^{R}\left(\left\{p_{j}\right\}\right)\right|^{2}-\mathscr{D}^{q_{1} g_{6}, \bar{q}_{2}}-\mathscr{D}^{\bar{q}_{2} g_{6}, q_{1}}\right] d \Phi_{V V V g},
$$

where the factor $1 / 6$ accounts for the three identical bosons in the final state. More details can be found in $[15,11]$.

The hadronic differential cross section with hadron momenta $P_{1}$ and $P_{2}$ is the sum over all partonic initial states convoluted with the parton distribution functions

$$
d \sigma\left(P_{1}, P_{2}\right)=\sum_{a b} \int d z_{1} d z_{2} f_{a}\left(z_{1}, \mu_{F}\right) f_{b}\left(z_{2}, \mu_{F}\right) d \sigma_{a b}\left(z_{1} P_{1}, z_{2} P_{2}\right)
$$

where the sum runs over the partonic configurations $q \bar{q}, \bar{q} q, g q, q g, g \bar{q}, \bar{q} g$.

As an explicit example we present the numerical results for the case $u \bar{u} \rightarrow Z Z Z$ for $\sqrt{s}=14 \mathrm{TeV}$ and using CTEQ6L1[16]. Tree-order cross section has been evaluated using the HELAC event generator[17]. In the following table the results in $\mathrm{fb}$ are presented for the tree-order cross section $\sigma_{0}$, the ratio of the virtual to the tree-level cross section, and the real contribution, combining 5and 6-point contributions, as described above, for all channels, i.e., $u \bar{u}, u g, g \bar{u}$, for different values of the factorization(renomralization) scale $\left(\mu=\mu_{F}=\mu_{R}\right)$.

\begin{tabular}{|c|c|c|c|c|}
\hline scale & $\sigma_{0}$ & $\sigma_{V} / \sigma_{0}$ & $\sigma_{R}$ & $\sigma_{N L O}$ \\
\hline$\mu=M_{Z}$ & $1.481(5)$ & $0.536(1)$ & $0.238(2)$ & $2.512(2)$ \\
$\mu=2 M_{Z}$ & $1.487(5)$ & $0.481(1)$ & $0.232(2)$ & $2.434(2)$ \\
$\mu=3 M_{Z}$ & $1.477(5)$ & $0.452(1)$ & $0.232(2)$ & $2.376(2)$ \\
$\mu=4 M_{Z}$ & $1.479(5)$ & $0.436(1)$ & $0.232(2)$ & $2.355(2)$ \\
$\mu=5 M_{Z}$ & $1.479(5)$ & $0.424(1)$ & $0.237(2)$ & $2.343(2)$ \\
\hline
\end{tabular}

As is evident from these results, the $K$-factor is quite sizeable $(1.58-1.69)$, whereas the dependence on the scale $\mu$ is for both cases quite weak, due mainly to the electroweak character of the process.

\section{Conclusions}

In conclusion we have presented and successfully tested in a number of applications, a reduction method at the integrand level that is changing the way we are looking at the NLO calculations: a full numerical but still algebraic method has been born. 
The efficiency of the OPP is quite good, the main factor being the efficiency with which the one-loop amplitude, at the integrand level, is computed.

Finally, taking into account the speed, precision and easiness of the OPP method, a universal NLO calculator/event-generator is feasible.

\section{References}

[1] G. Ossola, C. G. Papadopoulos and R. Pittau, Nucl. Phys. B 763 (2007) 147 [arXiv:hep-ph/0609007] and JHEP 0707 (2007) 085 [arXiv:0704.1271 [hep-ph]].

[2] F. del Aguila and R. Pittau, JHEP 0407 (2004) 017 [arXiv:hep-ph/0404120].

[3] Z. Bern, L. J. Dixon, D. C. Dunbar and D. A. Kosower, Nucl. Phys. B 435 (1995) 59 [arXiv:hep-ph/9409265].

[4] R. Britto, F. Cachazo and B. Feng, Nucl. Phys. B 725 (2005) 275 [arXiv:hep-th/0412103].

[5] W. B. Kilgore, arXiv:0711.5015 [hep-ph] and references therein;

R. K. Ellis, W. T. Giele and Z. Kunszt, arXiv:0708.2398 [hep-ph] and references therein.

[6] G. Ossola, C. G. Papadopoulos and R. Pittau, arXiv:0711.3596 [hep-ph].

[7] G. Ossola, C. G. Papadopoulos and R. Pittau, in preparation.

[8] R. Pittau, Comput. Phys. Commun. 104, 23 (1997) [arXiv:hep-ph/9607309] and 111 (1998) 48 [arXiv:hep-ph/9712418].

[9] D. H. Bailey; ARPREC (C++/Fortran-90 arbitrary precision package)

http://crd.lbl.gov/ dhbailey/mpdist/.

See also D. H. Bailey, "A Fortran-90 Based Multiprecision System," ACM Transactions on

Mathematical Software, vol. 21, no. 4 (Dec 1995), pg. 379-387.

[10] A. Lazopoulos, K. Melnikov and F. Petriello, Phys. Rev. D 76 (2007) 014001

[arXiv:hep-ph/0703273].

[11] T. Binoth, G. Heinrich, G. Ossola, C. G. Papadopoulos and R. Pittau, in preparation.

[12] A. van Hameren, J. Vollinga and S. Weinzierl, Eur. Phys. J. C 41 (2005) 361 [arXiv:hep-ph/0502165].

[13] W. T. Giele, E. W. N. Glover and D. A. Kosower, Nucl. Phys. B 403 (1993) 633 [arXiv:hep-ph/9302225].

[14] B. W. Harris and J. F. Owens, Phys. Rev. D 65 (2002) 094032 [arXiv:hep-ph/0102128].

[15] S. Catani and M. H. Seymour, Nucl. Phys. B 485 (1997) 291 [Erratum-ibid. B 510 (1998) 503] [arXiv:hep-ph/9605323].

[16] J. Pumplin, D. R. Stump, J. Huston, H. L. Lai, P. Nadolsky and W. K. Tung, JHEP 0207 (2002) 012 [arXiv:hep-ph/0201195].

[17] A. Kanaki and C. G. Papadopoulos, Comput. Phys. Commun. 132 (2000) 306 [arXiv:hep-ph/0002082].

A. Kanaki and C. G. Papadopoulos, arXiv:hep-ph/0012004.

A. Cafarella, C. G. Papadopoulos and M. Worek, arXiv:0710.2427 [hep-ph]. 